\title{
Combining the data of annual modulation effect in WIMP direct detection with measurements of WIMP indirect searches
}

\author{
A. Bottino ${ }^{\mathrm{a}, 1}$, F. Donato ${ }^{\mathrm{a}, 2}$, N. Fornengo ${ }^{\mathrm{a}, 3}$, S. Scopel ${ }^{\mathrm{b}, 4}$ \\ a Dipartimento di Fisica Teorica. Università di Torino and INFN, Sezione di Torino, Via P. Giuria I, lo125 Torino. Italy \\ ${ }^{\mathrm{h}}$ Instituto de Física Nuclear y Altas Energías, Facultad de Ciencias, Universidad de Zaragoza. Plaza de San Francisco s/n. \\ 50009 Zaragoza. Spain
}

Received 10 September 1998; accepted 2 October 1998

\begin{abstract}
In previous papers we showed that the data of the DAMA/NaI experiment for WIMP direct detection, which indicate a possible annual modulation effect, are widely compatiblc with an interpretation in terms of a relic ncutralino as the major component of dark matter in the Universe. In the present note we discuss the detectability of the relevant supersymmetric neutralino configurations by two of the most promising methods of indirect search for relic particles: measurement of cosmic-ray antiprotons and measurement of neutrino fluxes from Earth and Sun. (c) 1999 Elsevier Science B.V.
\end{abstract}

PACS: $11.30 . \mathrm{Pb} ; 12.60 . \mathrm{Jv} ; 95.35 .+\mathrm{d}$

\section{Introduction}

In Refs. [1-3] we showed that the indication of a possible annual modulation effect in WIMP direct search $[4,5]$ are interpretable in terms of a relic neutralino which may make up the major part of dark matter in the Universe.

We recall that the DAMA/NaI data [5] single out a very delimited $2 \sigma$ C.L. region in the plane $\xi \sigma_{\text {scalar }}^{\text {(nucleon) }}$ $m_{\chi}$, where $m_{X}$ is the WIMP mass, $\sigma_{\text {scalar }}^{\text {(nucleon }}$ is the WIMP-nucleon scalar elastic cross section and $\xi=$ $\rho_{X} / \rho_{l}$ is the fractional amount of local WIMP density $\rho_{X}$ with respect to the total local dark matter density $\rho_{l}$. In Fig. 1 we display the region $R$, which is obtained

\footnotetext{
'E-mail: bottino@to.infn.it

2 E-mail: donato@to.infn.it

3.E-mail: fornengo@to.infn.it

${ }^{4}$ INFN post-doctoral fellow; e-mail: scopel@posta.unizar.es
}

from the original $2 \sigma$ C.L. region of Ref. [5] (where $\rho_{l}$ is normalized to the value $\rho_{l}=0.3 \mathrm{GeV} \mathrm{cm}^{-3}$ ), by accounting for the uncertainty in the valuc of $\rho_{l}$. In obtaining region $R$ we have conservatively considered the range $0.1 \mathrm{GeV} \mathrm{cm}^{-3} \leq \rho_{l} \leq 0.7 \mathrm{GeV} \mathrm{cm}^{-3}$, because of a possible flattening of the dark matter halo $[6,7]$ and a possibly sizeable baryonic contribution to the galactic dark matter [8]. In Fig. 1 is also displayed the scatter plot of the supersymmetric configurations which, in our scanning of the susy parameter space, turn out to be contained in region $R$ (the set comprised of these configurations is called set $S$ ). The theoretical framework adopted here is the Minimal Supersymmetric extension of the Standard Model (MSSM), as illustrated in Refs. [1,2], to which we refer for details.

In [1-3] we have proved that a significant fraction of configurations of set $S$ is explorable at accelerators 


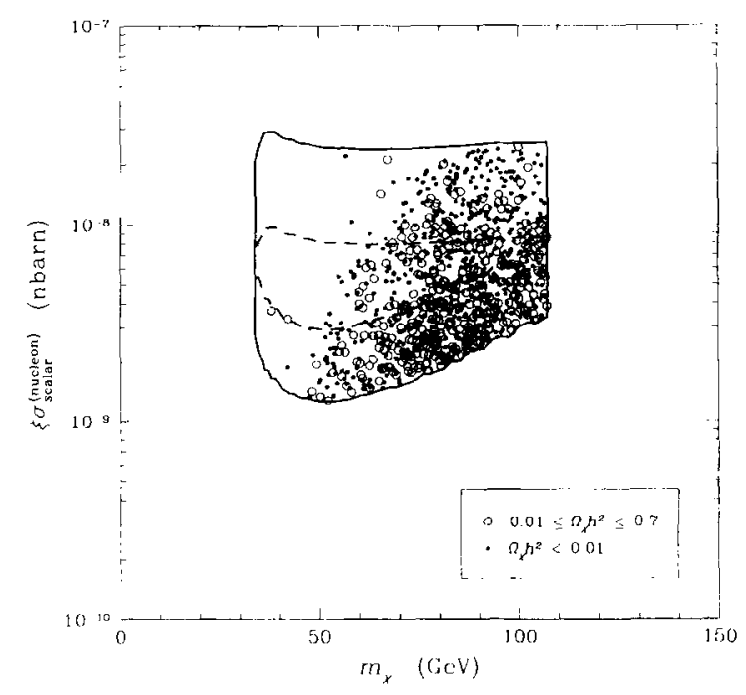

Fig. 1. Scatter plot of set $S$ in the plane $m_{\chi}-\xi \sigma_{\text {scalar }}^{\text {(nucleon) }}$. The dashed contour line delimits the $2 \sigma$ C.L. region, obtained by the DAMA/Nal Collaboration, by combining together the data of the two running periods of the annual modulation experiment [5]. The solid contour line is obtained from the dashed line, which refers to the value $\rho_{l}=0.3 \mathrm{GeV} \mathrm{cm} \mathrm{cm}^{-3}$, by accounting for the uncertainty range of $\rho$, as explained in Section 1 (the region delimited by the solid line is denoted as region $R$ in the text). Displayed in this figure are only the representative points of the susy parameter space, which fall inside the region $R$ (this set of configurations is defined as set $S$ ). Dots and circles denote neutralino configurations depending on their values for the neutralino relic abundance.

in the near future. In the present paper, we discuss the detectability of these configurations by different means, i.e. by two of the most promising ways of indirect search for relic particles: measurement of cosmicray antiprotons [9-11] and measurement of neutrino fluxes from Earth and Sun [12-14].

Preliminary discussions on the links between the possible annual modulation effect and the results of indirect searches have been presented in Refs. [1,11], in connection with the former DAMA/NaI data (data collected in the running period \# 1) [4]. Here we extend our investigation to the new results of the DAMA/NaI experiment (running period \# 2) [5], which establish a further indication for the annual modulation effect and, combined with the former ones, single out an effect at $99.6 \%$ C.L. In the present paper we also discuss neutralino cosmological properties in terms of its relic abundance $\Omega_{X} h^{2}$. This quantity is evaluated here as illustrated in [15], and for the parameter $\xi$ we take $\xi=\min \left(\Omega_{X} h^{2} /\left(\Omega h^{2}\right)_{\min }, 1\right)$, with $\left(\Omega h^{2}\right)_{\min }=0.01$. In exploring our supersymmetric parameter space we apply an upper bound, conservatively set at the value $\Omega_{\chi} h^{2} \leq 0.7$, and we consider as cosmologically interesting the range $0.01 \leq \Omega_{X} h^{2} \leq 0.7$. However, we stress that, according to the most recent data and analyses [16], the most appealing interval for the neutralino relic abundance is $0.02 \lesssim \Omega_{X} h^{2} \lesssim 0.2$.

Most of our results for the various quantities considered in this paper will be provided in the form of scatter plots, obtained by varying the representative points of the susy parameter space over the configurations of set $S$. When in a specific figure (or in a panel of it) the value of $\rho_{l}$ is fixed, it is meant that the corresponding scatter plot concerns only the subset of $S$ comprised of those configurations which belong to that particular value of $\rho_{l}$. For instance, for $\rho_{l}=0.3 \mathrm{GeV} \mathrm{cm}^{-3}$, only the points inside the dashed curve of Fig. 1 are considered.

\section{Cosmic-ray antiprotons}

Relic neutralinos, if present in our halo, would produce antiprotons by pair annihilation [9-11]. To discriminate this potential source of primary cosmic-ray $\bar{p}$ 's from the secondary antiprotons, i.e. $\bar{p}$ 's created by interactions of primary cosmic-ray nuclei with the interstellar medium, one can use the different features of their low-energy spectra $\left(T_{\bar{p}} \lesssim 1 \mathrm{GeV}, T_{\bar{p}}\right.$ being the antiproton kinetic-energy). In fact, in this energy regime the secondary $\bar{p}$ spectrum drops off very markedly because of kinematical reasons, while antiprotons due to neutralinos would show a milder fall off.

In Ref. [11] the low-energy cosmic-ray $\bar{p}$ spectrum has been calculated, in terms of a possible contribution of cosmic-ray antiprotons due to neutralino annihilation in the galactic halo and of a newly calculated flux for secondary antiprotons. The total spectrum has been compared with the data collected by the balloon-borne BESS spectrometer during its flight in 1995 (hereafter referred to as BESS95 data) [17]. The BESS95 data consist of 43 antiprotons grouped in 5 narrow energy windows over the total kinetic-energy range $0.180 \mathrm{GeV} \leq T_{\bar{p}} \leq 1.4 \mathrm{GeV}$.

In the present paper we evaluate the cosmic-ray antiproton flux as in Ref. [11], restricting the supersymmetric configurations to those of set $S$ and we com- 
pare our predictions with the BESS95 data. We refer to $[11]$ for all the details pertaining the evaluation of the production of primary and secondary antiprotons as well as for the properties related to their propagation in the halo and in the heliosphere. Here we only recall the features of the neutralino mass distribution function adopted in [11] as well as here. This mass distribution function is taken spheroidal and parameterized as a function $\rho_{\chi}(r, z)$ of the radial distance $r$ from the galactic center in the galactic plane and of the vertical distance $z$ from the galactic plane in the form

$\rho_{X}(r, z)=\rho_{X} \frac{a^{2}+r_{\odot}^{2}}{a^{2}+r^{2}+z^{2} / f^{2}}$,

where $a$ is the core radius of the halo, $r_{\odot}$ is the distance of the Sun from the galactic center and $f$ is a parameter which describes the flattening of the halo. Here we take the values $a=3.5 \mathrm{kpc}, r_{\odot}=8 \mathrm{kpc}$. The quantity $\rho_{\chi}$ denotes the local value of the neutralino matter density, which is factorized as $\rho_{X}=\xi \rho_{l}$, in terms of the total local dark matter density $\rho_{l}$. The density $\rho_{l}$ is calculated by taking into account the contribution given by the matter density of Eq. (1) to the local rotational velocity. We use here the value $\rho_{l}=$ $0.3 \mathrm{GeV} \mathrm{cm}^{-3}$ in the case of a spherical halo $(f=1)$. When $f<1$ (oblate spheroidal distribution), $\rho_{l}$ is given by $[18,7]$

$\rho_{l}(f)=\rho_{l}(f=1) \frac{\sqrt{1-f^{2}}}{f \arcsin \sqrt{1-f^{2}}}$.

Our results will be shown for the following values of the local density: $\rho_{l} /\left(\mathrm{GeV} \mathrm{cm}^{-3}\right)=0.1(f=1)$, $0.3(f=1), 0.5(f=0.50), 0.7(f=0.33)$. For each value of $\rho_{i}$, we will give the top-of-atmosphere (TOA) antiproton fluxes, as the sum of the secondary flux and of the primary flux due to neutralino annihilation for the various supersymmetric configurations of set $S$, pertaining to that specific value of $\rho_{l}$.

Fig. 2 displays the scatter plots for the top-ofatmosphere (TOA) antiproton fluxes calculated at $T_{\bar{p}}=0.24 \mathrm{GeV}$, to conform to the energy range of the first bin of the BESS95 data $\left(0.175 \mathrm{GeV} \leq T_{i} \leq\right.$ $0.3 \mathrm{GeV}$ ). This figure also shows the band of the experimental results in this bin. We find that, while most of the susy configurations of the appropriate subset of $S$ stay inside the experimental band for $\rho_{l}=0.1$, $0.3 \mathrm{GeV} \mathrm{cm}^{-3}$, at higher values of $\rho_{l}$ a large number

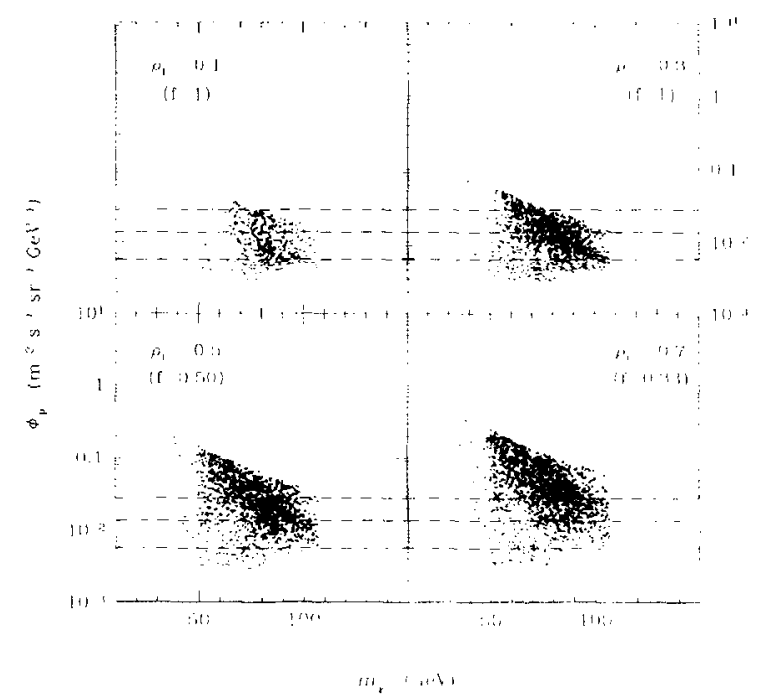

Fig. 2. Scatter plots for the TOA antiproton fluxes calculated at $T_{\bar{p}}=0.24 \mathrm{GeV}$ versus the neutralino mass. The configurations of set $S$ are subdivided into the 4 panels. depending on the corresponding value of the local density: $\rho_{l} /(\mathrm{GeV} \mathrm{cm}-3)=0.1$ $(f=1), 0.3(f=1), 0.5(f=0.50), 0.7(f=0.33)$. The dashed lines denote the central value and the $1 \sigma \sigma$ band of the BESS95 data in the first energy bin: $0.175 \mathrm{GcV} \leq T_{\bar{p}} \leq 0.3 \mathrm{GeV}$.

of configurations provide $\bar{p}$ fluxes in excess of the experimental results. This occurrence is easily understood on the basis of the different dependence on $\rho_{l}$ of the direct detection rate and of the $\bar{p}$ flux, linear in the first case and quadratic in the second one.

To present the comparison of our theoretical fluxes with the experimental ones in a more comprehensive way, we give in Fig. 3 the scatter plots of the reduced chi-square $\chi_{\text {red }}^{2}$ values, obtained for the configurations of each of the four subsets of $S$, in a fit to the BESS data over the whole energy range $\left(0.175 \mathrm{GeV} \leq T_{\bar{p}} \leq\right.$ $1.4 \mathrm{GeV}$ ). In these scatter plots we have included only configurations which are at least at the level of the experimental value (within $1 \sigma$ ) in the first energy bin. We remark that, apart from values of $\rho_{l}$ close to the largest value of its physical range, a large sample of the susy configurations, relevant for annual modulation data in direct search of relic neutralinos, provides a good fit to the current data on cosmic antiprotons.

By way of example, we show in Fig. 4 a sample of fluxes obtained with a few susy configurations of set $S$ which give a good fit to the BESS95 data. The parameter $P$, which denotes the various supersymmetric configurations, is the gaugino fractional weight (for 


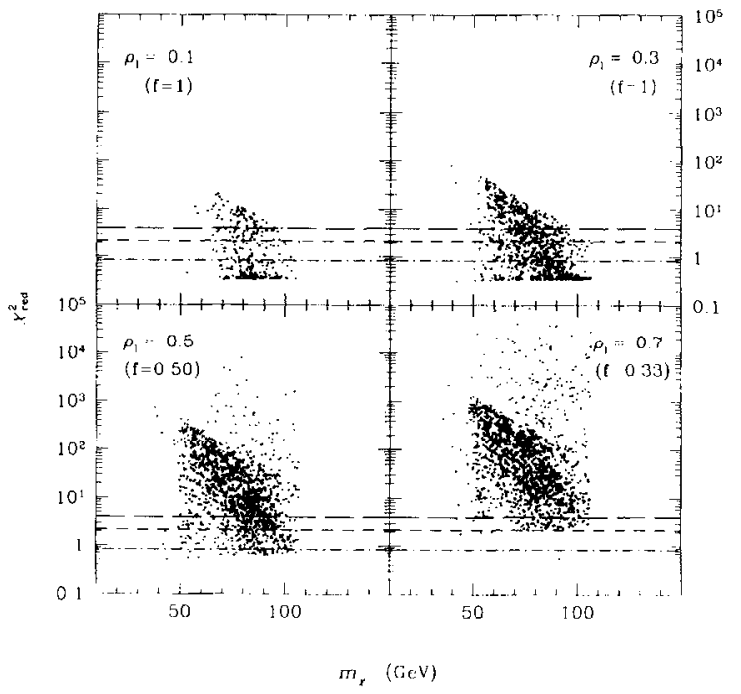

Fig. 3. Scatter plots of the $\chi_{\text {red }}^{2}$ values, obtained for the configura. tions of each of the four subsets of $S$, in a fit to the BESS95 data over the whole energy range $\left(0.175 \mathrm{GeV} \leq T_{\bar{p}} \leq 1.4 \mathrm{GeV}\right)$ versus the neutralino mass. Only configurations which are at least at the level of the experimental value (within $1 \sigma$ ) in the first energy bin are included. The three horizontal lines represent the $\chi_{\text {red }}^{2}$ in a fit of the data using the secondary flux only (dot-dashed line), and the $\chi_{\text {red }}^{2}$ in a fit using the total (primary plus secondary) flux at $95 \%$ C.L. (short-dashed line) and at $99.9 \%$ C.L. (long-dashed line). The $\rho_{l}$ values are in units of $\mathrm{GeV} \mathrm{cm}{ }^{-3}$.

instance, a neutralino which is a pure gaugino has $P=$ 1).

From Fig. 3 it also turns out that, especially at large $\rho_{l}$, many configurations of set $S$ have very large values of $\chi_{\text {red }}^{2}$. Configurations which have $\chi_{\text {red }}^{2}>4$ may be considered as strongly disfavoured on the basis of the present experimental data on cosmic antiprotons ( $\chi_{\text {red }}^{2}=4$ corresponds to a $99.9 \%$ C.L. in our case of 5 degrees of freedom ). However, because of the many uncertainties involved in the current theoretical evaluations (for instance, effects due to solar modulation of the original interstellar fluxes) and in the experimental measurements of the antiproton spectrum, a strict exclusion criterion may be applied only to configurations which are in large excess of $\chi_{\text {red }}^{2}=4$. By the same arguments, from our results we derive that high values of $\rho_{l}$, i.e. $\rho_{l} \sim 0.7 \mathrm{GeV} \mathrm{cm}{ }^{-3}$, appear to be disfavoured. Hereafter, configurations with $\chi_{\text {red }}^{2}>4$ will be denoted by special symbols.

In Fig. 5 we give $\chi_{\text {red }}^{2}$ versus the neutralino relic abundance. On the basis of these results, it turns out

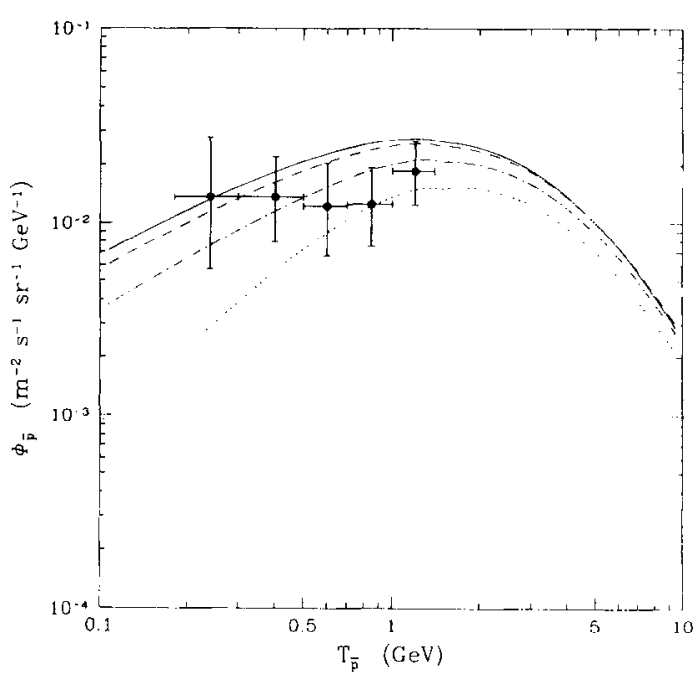

Fig. 4. Some examples of TOA fluxes which provide a good fit to the BESS95 data, for $\rho_{l}=0.3 \mathrm{GeV} \mathrm{cm}{ }^{-3}$. The dotted line denotes the secondary antiproton flux only. The solid, dashed and dot-dashed lines denote the total antiproton fluxes, obtained as a sum of the secondary spectrum and of the primary spectra due, respectively, to the following neutralino configurations: $m_{\chi}=62 \mathrm{GeV}, \Omega_{\chi} h^{2}=0.03$ and $P=0.98$ (solid line); $m_{\chi}=81 \mathrm{GeV}, \Omega_{\chi} h^{2}=0.02$ and $P=0.99$ (dashed line); $m_{\chi}=95 \mathrm{GeV}, \Omega_{\chi} h^{2}=0.03$ and $P=0.99$ (dot-dashed line).

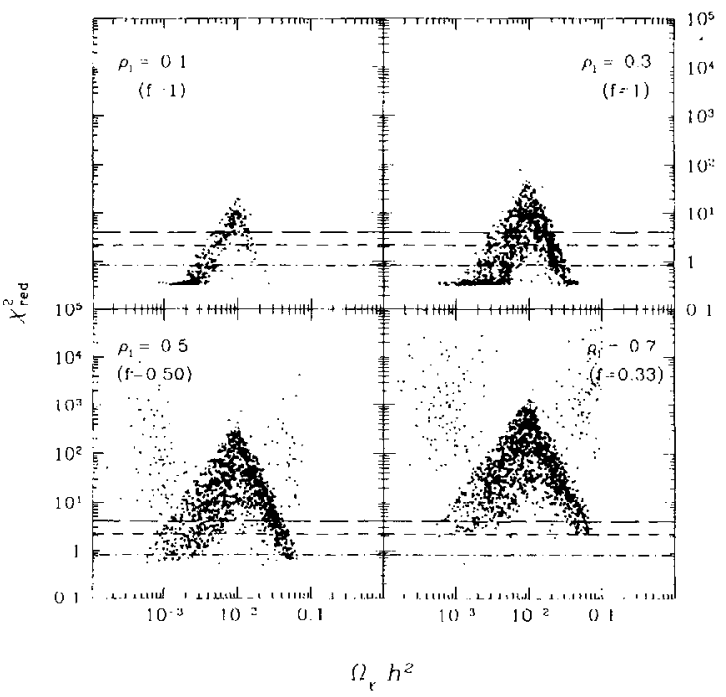

Fig. 5. Scatter plots of the $\chi_{\text {red }}^{2}$ values, obtained for the configurations of each of the four subsets of $S$, in a fit to the BESS data over the whole energy range $\left(0.175 \mathrm{GeV} \leq T_{\bar{p}} \leq 1.4 \mathrm{GeV}\right)$ versus the neutralino relic abundance. Notations as in Fig. 3 . 
that the central values of the range for the local density. $0.3 \mathrm{GeV} \mathrm{cm}^{-3} \lesssim \rho_{l} \lesssim 0.5 \mathrm{GeV} \mathrm{cm}^{-3}$, is the best suited for neutralinos of cosmological interest. The break at $\Omega_{x} h^{2}=0.01$ in the shape of the scatter plots in this figure is due to the rescaling in the local density introduced through the parameter $\xi$ for $\Omega_{\chi} h^{2} \leq$ $\left(\Omega h^{2}\right)_{\min } \equiv 0.01$. Because of this, one roughly has $\Phi_{j ;} \times\left(\sigma_{\mathrm{ann}}\right)^{-1} \times \Omega_{\chi} h^{2}$ for $\Omega_{\chi} h^{2} \leq\left(\Omega h^{2}\right)_{\min }$, and $\Phi_{\tilde{p}} \propto \sigma_{\mathrm{ann}} \propto\left(\Omega_{\chi} h^{2}\right)^{-1}$ otherwise, where $\sigma_{\mathrm{ann}}$ is the neutralino-pair annihilation cross section.

The various results discussed in this section show the remarkable property that a number of the supersymmetric configurations singled out by the annual modulation data may indeed produce measurable effects in the low-energy part of the $\bar{p}$ spectrum. Thus the joint use of the annual modulation data in direct detection and of the measurements of cosmic-ray antiprotons is extremely uscful in pinning down a number of important properties of relic neutralinos and show the character of complementarity of these two classes of experimental searches for particle dark matter. This stresses the great interest for the analyses now under way of new antiproton data, those collected by a recent balloon flight carried out by the BESS Collaboration [24] and those measured by the AMS experiment [25] during the June 1998 Shuttle flight.

\section{Neutrinos from Earth and Sun}

The signals we are going to discuss now consist of the fluxes of up-going muons in a neutrino telescope, generated by neutrinos which are produced by pair annihilations of neutralinos captured and accumulated inside the Earth and the Sun [12-14]. The process goes through the following steps: (a) capture by the celestial body of the relic neutralinos through a slowdown process due essentially to neutralino elastic scattering off the nuclei of the macroscopic body, (b) accumulation of the captured neutralinos in the central part of the celestial body, (c) neutralino-neutralino annihilation with emission of neutrinos, (d) propagation of neutrinos and conversion of their $\nu_{\mu}$ component into muons in the rock surrounding the detector (or, much less efficiently, inside the detector), and finally (e) propagation and detection of the ensuing up-going muons in the detector.

The various quantities relevant for the previous steps are calculated here according to the method described in Ref. [13,14], to which we refer for further details. Here we only wish to recall that the neutrino fluxes are proportional to the annihilation rate of the neutralinos inside the macroscopic body, $\Gamma_{A}$, which is given by [19]

$\Gamma_{A}=\frac{C}{2} \tanh ^{2}\left(\frac{t}{\tau_{A}}\right)$.

where $t$ is the age of the macroscopic body $(t \simeq$ $4.5 \mathrm{Gyr}$ for Sun and Earth) and $\tau_{A}=\left(C C_{A}\right)^{-1 / 2} ; C_{A}$ is the annihilation rate proportional to the neutralinoneutralino annihilation cross section and $C$ is the capture rate, proportional to the neutralino-nucleus cross section and to the neutralino local density. From Eq. (3) it follows that in a given macroscopic body the equilibrium between capture and annihilation (i.e. $\Gamma_{A} \sim C / 2$ ) is established only when $t \geq \tau_{A}$.

From the evaluation of the annihilation rate for neutralinos inside the Earth and the Sun it turns out that. for the Earth, the equilibrium condition depends critically on the values of the model parameters, and is not realized in wide regions of the parameter space. Consequently, for these regions the signal due to neutralino annihilation may be significantly attenuated. Capture by the Earth is very efficient for $m_{\chi} \lesssim 70 \mathrm{GeV}$ because of the mass-matching condition between the neutralino mass and the nuclear mass of some important chemical constituents of the Earth $(\mathrm{O}, \mathrm{Si}, \mathrm{Mg}, \mathrm{Fe})$. On the contrary, in the case of the Sun, the capture-annihilation equilibrium is reached for the whole range of $m_{\chi}$, due to the much more efficient capture rate due to thc stronger gravitational field [20].

Let us now report our results. In Fig. 6 we display the scatter plots for the flux of the up-going muons from the center of the Earth, for various values of the local dark matter density $\rho_{l}$. In this figure is also reported the current $90 \%$ C.L. experimental upper bound on $\Phi_{\mu}^{\text {Earth }}$, obtained by MACRO [21] (a similar experimental upper bound is given in Ref. [22]). We note the particular enhancement at $m_{X} \sim(50-60) \mathrm{GeV}$, due to the mass-matching effect. It is also noticeable an effect of suppression and spreading of the fluxes for $m_{X} \gtrsim 70 \mathrm{GeV}$ at $\rho_{l} \gtrsim 0.3 \mathrm{GeV} \mathrm{cm}{ }^{-3}$. This is due to the fact that the configurations with these large values of $\rho_{l}$ may imply, because of the annual modulation data, a neutralino-nucleus cross section which is too small to establish an efficient capture rate, neces- 


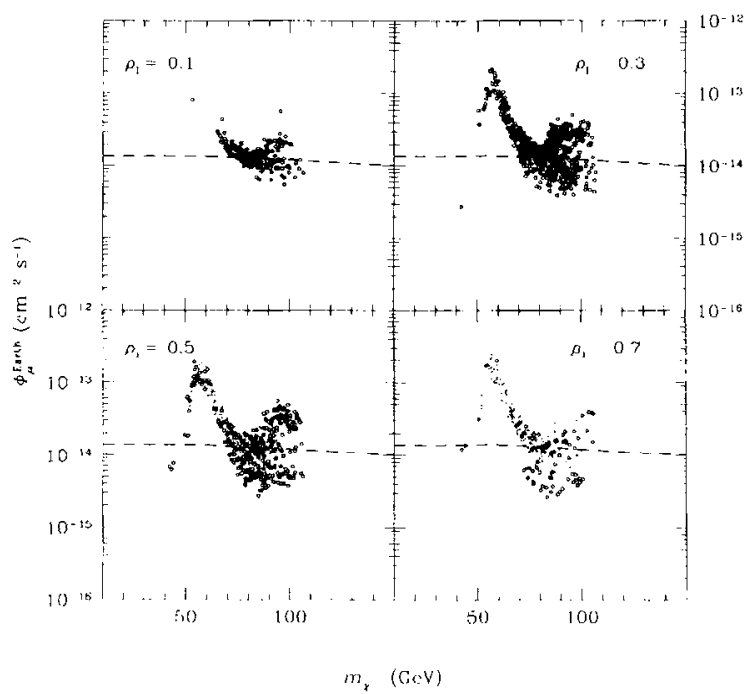

Fig 6. Scatter plots for the up-going muon fluxes from the center of the Earth versus the neutralino mass. The configurations of set $S$ are subdivided into the 4 panels, depending on the corresponding value the local density: $\rho_{/} /\left(\mathrm{GeV} \mathrm{cm}^{-3}\right)=0.1(f=1), 0.3(f=1)$, $0.5(f=0.50), 0.7(f=0.33)$. Dots denote configurations which could be excluded on the basis of the BESS95 antiproton data, circles denote configurations which survive this exclusion criterion. The dashed line denotes the MACRO upper bound.

sary for the capture-annihilation equilibrium in Earth. This is shown in Fig. 7, whose scatter plots show that indeed in the case of large values of $\rho_{l}$ many configurations induce sizeable deviations from the value $\tanh ^{2}\left(t / t_{A}\right)=1$, which guarantees equilibrium in the macroscopic body.

In Fig. 6 the configurations which would be excluded on the basis of the antiproton data, as previously discussed in Section 2, are denoted differently from those which would survive this criterion.

By comparing our scatter plots with the experimental MACRO upper limit, one notices that a number of supersymmetric configurations provide a flux in excess of this experimental bound and might then be considered as excluded. However, it has to be recalled that a possible neutrino oscillation effect [23] may be operative here and affect the indirect neutralino signal as well as the background consisting in atmospheric neutrinos. The influence of a neutrino oscillation on the problem under discussion would depend very critically on the nature of the oscillation itself ( $\nu_{\mu}$ converting either into a $\nu_{\tau}$ or into a sterile neutrino). Therefore, a strict enforcement of the current

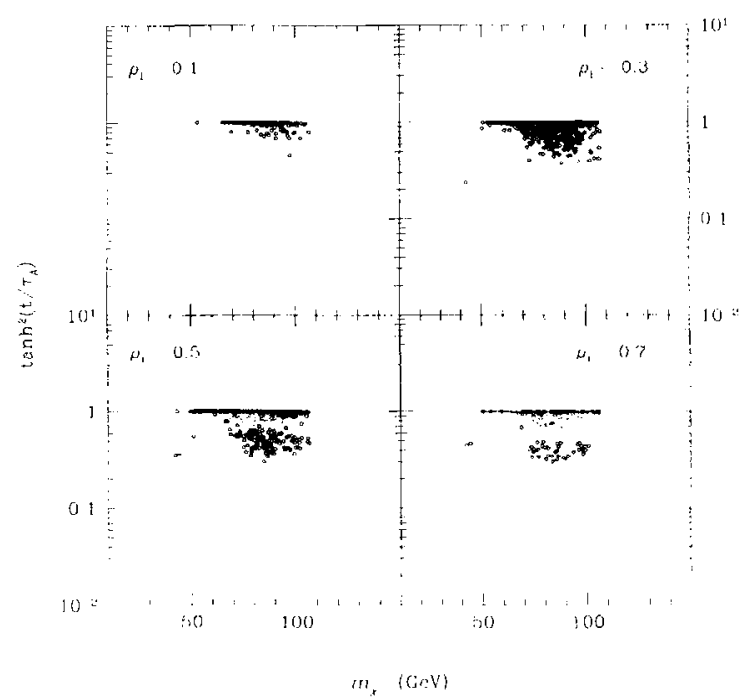

Fig. 7. Scatter plots of the quantity $\tanh ^{2}\left(t / \tau_{A}\right)$ versus the neu tralino mass. Other specifications are as in Fig. 6.

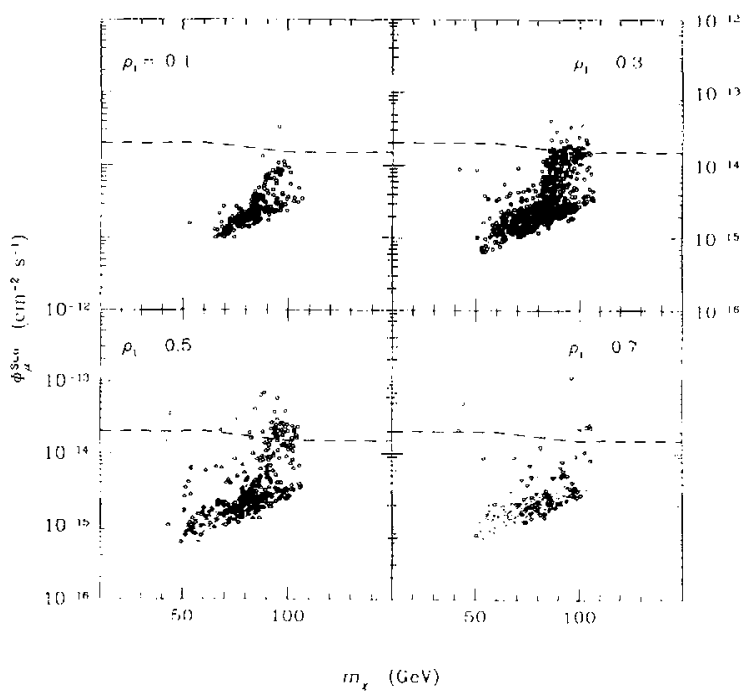

Fig. 8. Scatter plots for the up-going muon fluxes from the Sun versus the neutralino mass. Other specifications are as in Fig. 6.

upper bound on $\Phi_{\mu}^{\text {Earth }}$ should be applied with caution as long as the neutrino oscillation properties are not fully understood. However, it is rewarding that the set $S$ of supersymmetric configurations is quite accessible to relic neutralino indirect search by measurements of up-going fluxes, as displayed in our results of Fig. 6.

Fig. 8 displays the up-going muon fluxes from the Sun. We notice that a number of configurations of higher masses are easily accessible to the measure- 
ments of these fluxes.

\section{Conclusions}

We have proved that a sizeable fraction of the supersymmetric neutralino configurations singled out by the DAMA/NaI data on a possible annual modulation effect in WIMP direct search may provide signals detectable by two of the most interesting methods of indirect search for relic particles: measurement of cosmic-ray antiprotons and measurement of neutrino fluxes from Earth and Sun.

For the case of cosmic antiprotons, it has been shown that present data are well fitted by total spectra which include a $\bar{p}$ contribution from neutralinopair annihilation, with neutralino configurations which are relevant for annual modulation in direct detection. These data can also be used to reduce the total sample of the supersymmetric configurations under study, and to narrow the range of the local density, by disfavouring its largest values. Investigation by measurements of cosmic $\bar{p}$ 's looks very promising in view of the collections and analyses of more statistically significant sets of data in the low-energy regime which are currently under way $[24,25]$ and which may soon provide further relevant information.

Mcasurements of neutrino fluxes from Earth and Sun, due to capture and annihilation of neutralinos inside these celestial bodies, have been proved to be sensitive to neutralino configurations singled out by the annual modulation data. An appropriate interpretation of these measurements preliminarily requires some clarification of the oscillation neutrino propertics.

In conclusion, we have shown how the measurements of cosmic-ray antiprotons and of neutrino fluxes from Earth and Sun are complementary to those of direct detection in investigating astrophysical and cosmological properties of the relic neutralinos which may he at the origin of the possible modulation effect in direct search.

\section{References}

I1] A. Bottino, F. Donato, N. Fornengo, S. Scopel, Phys. Lett. B 423 (1998) 109.
[2] A. Bottino, F. Donato, N. Fornengo, S. Scopel, Torino University preprint DFTT 41/98, hep-ph/9808456, Phys. Rev. D, submitted.

[3] A. Bottino, F. Donato, N. Fornengo, S. Scopel, Torino University preprint DFTT 48/98, hep-ph/9808459. Phys. Rev. D, submitted.

|4| R. Bernabei et al.. Phys. Lett. B 424 (1998) 195

[5] R. Bernabei et al., Roma II University preprints: ROMA2F/98/27 and ROMA2F/98/34 (August 1998).

(6) E. Gates, G. Gyuk. M.S. Turner, Phys. Rev. Letr. 74 (1995) 3724; Phys. Rev. D 53 (1996) 4138

17| E. Gates, G. Gyuk, M.S. Turner. Astrophys. J. Lett. 449 (1995) L/23.

181 E. Gates, G. Gyuk, M.S. Turner, talk presented at: 18th Texas Symposium on Relativistic Astrophysics. Chicago. December 1996, astro-ph/9704253.

19| J. Silk, M. Srednicki. Phys. Rev. Lett. 53 (1984) 624: J. Eltis, R.A. Flores, K. Freese, S. Ritz, D. Seckel, J. Silk. Phys. Lett. B 214 (1988) 403;

F. Stecker, S. Rudaz. T. Walsch. Phys. Rev. Lett. 5,5 ( 1985) 2622 ;

J.S. Hagelin, G.L. Kane, Nucl. Phys. B 26.3 (1986) 399;

S. Rudaz, F.W. Stecker, Astrophys. J. 325 (1988) 16;

F. Stecker, A. Iylka, Astrophys. J. 336 ( I989) L. II;

G. Jungman, M. Kamionkowski, Phys. Rev. D 49 (1994) 2316

T. Mitsui, K. Maki. S. Orito, Phys. Lett. B 389 (1996) 169.

[10] A. Bottino, C. Favero, N. Fornengo, G. Mignola, Astropan. Phys. 3 (1995) 77.

I11] A. Bottino. F. Donato, N. Fornengo, P. Salati, Phys, Rev. D) 58 (1998) 123503.

[12] J. Silk, K. Olive. M. Srednicki, Phys. Rev. Lett. 55 (1985) 257:

T. Gaisser, G. Steigman, S. Tilav, Phys. Rev. D 34 (1986) 2206:

K. Freese, Phys. Lett. B 167 (1986) 295:

K. Griest, S. Seckel, Nucl. Phys. B 279 ( 1987) 804:

G.F. Giudice, E. Roulet, Nucl. Phys. B 316 (1989) 429:

G.B. Gelmini, P. Gondolo, E. Roulet, Nucl. Phys. B 351 (1991) 623;

M. Kamionkowski, Phys. Rev. D 44 (1991) 3021:

A. Bottino, $V$. de Alfaro, N. Fornengo, G. Mignola, M. Pignone, Phys. Lett. B 265 (1991) 57:

F. Halzen, M. Kamionkowski, T. Steltzer, Phys. Rev. D 45 (1992) 4439;

V.S. Berezinsky, Proc. Neutrino 92. A. Morales, ed. Nucl. Phys. (Proc. Suppl.) B 31 (1993) 413;

M. Mori et al., Phys. Rev. D 48 (1993) 5505:

M. Drees, G. Jungman, M. Kamionkowski, M.M. Nojiri, Phys. Rev. D 49 (1994) 636:

R. Gandhi, J.L. Lopez, D.V. Nanopoulos, K. Yuan, A. Zichichi, Phys. Rev. D 49 (1994) 3691 ;

L. Bergström, J. Edsjö, P. Gondolo, Phys. Rev. D 55 (1997) 1765:

L. Bergström, J. Edsjö, M. Kamionkowski, Astropart. Phys. 7 (1997) 147 
[13] A. Bottino, N. Fornengo, G. Mignola, L. Moscoso, Astropart. Phys. 3 (1995) 65.

[14] V. Berezinsky, A. Bottino, J. Ellis, N. Fornengo, G. Mignola, S. Scopel, Astropart. Phys, 5 (1966) 333.

[15] A. Bottino, V. de Alfaro, N. Fornengo, G. Mignola, M. Pignone, Astropart. Phys. 2 (1994) 67.

[16] W.L. Freedman, R. Kirshner, C. Lineweaver, talks given at: International Conference of Cosmology and Particle Physics, Geneva, June 1998, http://wwwth.cern.ch/ capp98/programme.html;

M. White, Astrophys. J. 506 (1998) 495;

N.A. Bahcall, X. Fan, astro-ph/9804082;

C. Lineweaver, astro-ph/9805326.

[17] H. Matsunaga et al., BESS Collaboration, in: Proc. 25th Int. Conference of Cosmic Rays, Durban, 1997;

A. Moiseev, private communication.

[18] J. Binney, S. Tremaine, Galactic Dynamics (Princeton University Press, 1987).
[19] K. Griest, D. Seckel, Nucl. Phys. B 283 (1987) 681.

[20] A. Gould, Astropart. J. 321 (1987) 571; 328 (1988) 919; 368 (1991) 610 .

[21] M. Ambrosio et al., MACRO Collabotation, MACRO/PUB$1 / 98$

T. Montaruli et al., Proceedings of TAUP97, Nucl. Phys. B 70 (Proc. Suppl.) (1999) 367.

[22] M.M. Boliev et al., Proceedings of TAUP97, Nucl. Phys. B 70 (Proc. Suppl.) (1999) 371.

[23] Y. Fukuda et al., Super Kamiokande Collaboration, hepex/9807003.

[24] S. Orito, talk at: Int. Conf. on High Energy Physics. Vancouver, July 1998.

[25] S. Ahlen et al., Nucl. Instrum. Meth. A 350 (1994) 351.

[26] R. Bernabei et al., Phys. Lett. B 389 (1996) 757. 